\title{
On geometric interpretation of the Aharonov-Bohm effect
}

\author{
M. O. Katanaev* \\ Steklov Mathematical Institute, \\ ul. Gubkina, 8, Moscow, 119991, Russia
}

December 11, 2012

\begin{abstract}
A geometric interpretation of the Aharonov-Bohm effect is given in terms of connections on principal fiber bundles. It is demonstrated that the principal fiber bundle can be trivial while the connection and its holonomy group are nontrivial. Therefore, the main role is played by geometric rather than topological effects.
\end{abstract}

\section{Introduction}

The Aharonov-Bohm effect [1] has attracted great attention of theorists and experimenters for many years. The interest is caused by the following circumstance. In the theory of gauge fields, the popular belief is that only nontrivial field strength rather than the potentials themselves that are not gauge-invariant can cause the observable effects. Contrary to this opinion, Aharonov and Bohm have demonstrated that the integral of a gauge field along a closed loop can produce the observable effects. The effect produced by the magnetic potential was soon confirmed experimentally [2, 3, 4].

In the present work, we give a geometric interpretation of the Aharonov-Bohm effect in terms of connections on the principal fiber bundle. Observable effects are produced by holonomy group elements for the corresponding connections. The elements of the holonomy group are gauge invariant because the group $\mathbb{U}(1)$ is Abelian one. Many authors relate the Aharonov-Bohm effect to the nontrivial topology of space. We here demonstrate that the principal fiber bundle can be trivial, while the connection arising on it generally has a nontrivial holonomy group and hence leads to the observable effects. From this it follows that the Aharonov-Bohm effect has geometric rather than topological nature.

The Aharonov-Bohm effect from the geometric viewpoint is similar to the Berry phase [5] (see also [6]). In both cases, the observable effects are produced by holonomy group elements. The difference is that bases of the corresponding principal fiber bundles are different manifolds. For the Aharonov-Bohm effect, the base is the four-dimensional space-time while for the Berry phase, it is a manifold of external parameters (for example, the range of variation of the external magnetic field) on which the Hamiltonian depends.

*E-mail: katanaev@mi.ras.ru 


\section{Aharonov-Bohm effect}

The Aharonov-Bohm effect [1] gives an example of occurrence of a nontrivial connection on the trivial principal fiber bundle $\mathbb{P}\left(\mathbb{R}^{4}, \pi, \mathbb{U}(1)\right) \approx \mathbb{R}^{4} \times \mathbb{U}(1)$ in nonrelativistic quantum mechanics. In this case, unlike the Berry phase, the space-time $\mathbb{R}^{4}$ in which the particle moves acts as the base $\mathbb{M}$ of the principal fiber bundle.

Let us consider the Schrödinger equation [7, 8]

$$
i \hbar \frac{\partial \psi}{\partial t}=H \psi
$$

where $\hbar$ is the Planck constant. Let the Hamiltonian describe the motion of a point particle of mass $m$ in the three dimensional Euclidean space $\mathbb{R}^{3}$ with the Cartesian coordinates $x^{\mu}, \mu=1,2,3$,

$$
H_{0}=-\frac{\eta^{\mu \nu} p_{\mu} p_{\nu}}{2 m}+U=-\frac{\hbar^{2}}{2 m} \triangle+U
$$

where $p_{\mu}=i \hbar \partial_{\mu}$ is the particle momentum operator, $\eta_{\mu \nu}=\operatorname{diag}(---)$ is the negative definite space metric, $\triangle=\partial_{1}^{2}+\partial_{2}^{2}+\partial_{3}^{2}$ is the Laplace operator, and $U(x)$ is the potential energy of the particle.

We write down the four dimensional momentum operator in the form $p_{\alpha}=i \hbar \partial_{\alpha}$, $\alpha=0,1,2,3$. In this case, the zero 4-momentum component $p_{0}=i \hbar \partial_{0}=i \hbar \partial_{t}$ has the physical meaning of the particle energy operator.

If a particle interacts with an electromagnetic field, this interaction is described by means of the minimal substitution for all four components of the momentum

$$
p_{\alpha} \mapsto i \hbar \partial_{\alpha}-\frac{e}{c} A_{\alpha}
$$

where $e$ is the particle charge, $c$ is the velocity of light, and $A_{\alpha}$ is the electromagnetic field potential (components of the local form of the $\mathbb{U}(1)$-connection). In this case, the zero component divided by the velocity of light, $A_{0} / c$, has the physical meaning of the electrical field potential, and the spatial components $A_{\mu}$ are the covector components of the magnetic field potential. Thus, the point particle moving in the electromagnetic field is described by the Schrödinger equation

$$
i \hbar \frac{\partial \psi}{\partial t}=\left[\frac{\hbar^{2}}{2 m} \eta^{\mu \nu}\left(\partial_{\mu}+i \frac{e}{\hbar c} A_{\mu}\right)\left(\partial_{\nu}+i \frac{e}{\hbar c} A_{\nu}\right)+\frac{e}{c} A_{0}\right] \psi+U \psi .
$$

For simplicity, we further put $\hbar=1$ and $c=1$.

From the geometric viewpoint, minimal substitution (3) coincides to within constants with replacement of the partial derivative by the covariant one:

$$
\partial_{\alpha} \mapsto \partial_{\alpha}+i e A_{\alpha}
$$

Let us consider Schrödinger equation (44) from the geometric viewpoint. It is solved in the whole space-time $\psi=\psi(t, x)$; therefore, the base of the fiber bundle (for example, see [9]) is the four-dimensional Euclidean space $(t, x) \in \mathbb{R} \times \mathbb{R}^{3}=\mathbb{R}^{4}$. In this case, $\mathbb{R}^{4}$ is considered simply as a four-dimensional manifold without any four-dimensional metric. If required, the metric can be introduced but its presence does not alter the principal fiber bundle and the connection. The metric $\eta_{\mu \nu}$ is defined only on spacelike sections $t=$ const, because it enters the Schrödinger equation. The wave function $\psi(t, x)$ is the section of the fiber bundle $\mathbb{E}\left(\mathbb{R}^{4}, \pi_{\mathbb{E}}, \mathbb{C}, \mathbb{U}(1), \mathbb{P}\right)$ with the typical fiber being the complex plane $\mathbb{C}$, which 
is associated with a certain principal fiber bundle $\mathbb{P}\left(\mathbb{R}^{4}, \pi, \mathbb{U}(1)\right)$. This principal fiber bundle is always trivial $\mathbb{P} \approx \mathbb{R}^{4} \times \mathbb{U}(1)$, because its base is the four-dimensional Euclidean space. The local $\mathbb{U}(1)$-connection form defined by the electromagnetic potential $A_{\alpha}(t, x)$, is set on this principal fiber bundle. In nonrelativistic quantum mechanics, instead of the whole set of sections of associated fiber bundles, we consider only the subset $\psi(t, x)$ consisting of such differentiable sections which belong to the Hilbert space of square integrable functions $\mathbb{H}=\mathcal{L}_{2}\left(\mathbb{R}^{3}\right)$ on spacelike sections $\mathbb{R}^{3}$ for each moment of time $t$.

We consider two cases.

\subsection{Electric potential}

Let us assume that the magnetic potential is equal to zero, $A_{\mu}=0, \mu=1,2,3$. We now write down the Schrödinger equation in the form

$$
i \dot{\psi}=\left(H_{0}+e A_{0}\right) \psi
$$

where $H_{0}$ is the Hamiltonian of the system without the electromagnetic potential (2), and the dot atop designates differentiation with respect to time. We also assume that the electric potential depends only on time, $A_{0}=A_{0}(t)$. We seek a solution of Schrödinger equation (5) in the form $\psi=\mathrm{e}^{-i \Theta} \phi$ where $\phi$ is the solution of the free Schrödinger equation:

$$
i \dot{\phi}=H_{0} \phi
$$

and $\Theta=\Theta(t)$ is a certain phase independent of the space point. Substitution of $\psi=\mathrm{e}^{-i \Theta} \phi$ into initial Schrödinger equation (5) leads to the equation for the phase

$$
\dot{\Theta}=e A_{0},
$$

where we have canceled the common phase multiplier $\mathrm{e}^{-i \Theta}$ and $\phi$. This can be done, because the Schrödinger equation must be fulfilled for all $t$ and $x$. The solution of this equation has the form

$$
\Theta(t)=\Theta_{0}+e \int_{0}^{t} d s A_{0}(s)
$$

where $\Theta_{0}$ is the value of the wave function phase at the initial moment of time.

Aharonov and Bohm proposed the experiment whose scheme is shown in Fig [1. The beam of electrons is split into two beams transmitted through two metal tubes to which different potentials are applied. Then beams are collected, and the interference pattern is observed on the screen. The electric potential applied to the tubes depends on time. It is supposed to be zero until both beams are inside the tubes. Then it increases to some values different for each tube, and then decreases to zero when beams leave the tubes. Thus, the beams are in the field $A_{0}$ only when they are inside the tubes.

The interference pattern depends on the electron phase difference in the beams, which can be estimated approximately as follows. Assume that an electron is described by the wave function $\psi(t, x)$ which satisfies Schrödinger equation (5) in the whole space-time $\mathbb{R}^{4}$. We consider that at each moment of time, the wave function support is nonzero in a small neighborhood of space near the particle trajectory. Only in this case it is possible to speak about the particle trajectory. In particular, when the electron passes through the metal tube, it is assumed that the support of the wave function lies completely in the tube. Mathematically, this can be described by the corresponding potential introduced into Schrödinger equation (5). This hypothetical potential does not change the spacetime, that is, the base of the principal fiber bundle; it only provides the electron motion 


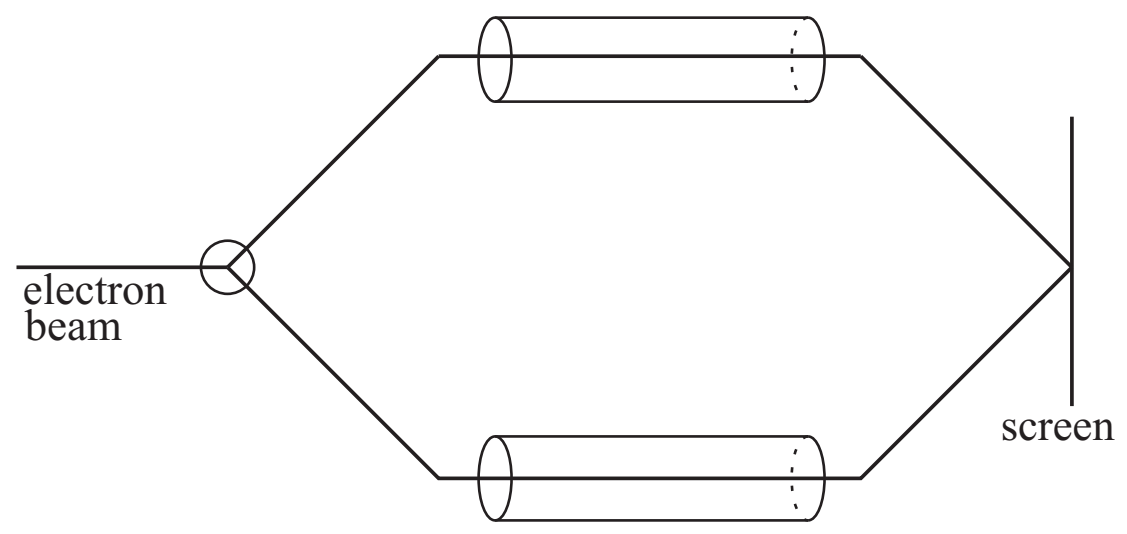

Figure 1: Scheme of the Aharonov-Bohm experiment.

along the present trajectory. We now estimate the electron phase change in the upper beam. Since the electric field potential is homogeneous inside the tube and the support of the wave function is completely localized inside the tube, we can consider that the phase of the wave function is defined by integral (7). We designate the moments of time the beam splits and achieves the screen by $t_{1}$ and $t_{2}$, respectively. Then the phase of the electron wave function of the upper beam, when it approaches the screen, changes by the value determined by the integral:

$$
\Theta_{1}=e \int_{t_{1}}^{t_{2}} d t A_{0}^{(1)}(t),
$$

where $A_{0}^{(1)}(t)$ is the electric field potential at the moment of time $t$, that is, at that point of space where the electron of the upper beam is located at the moment of time $t$. Analogously, the phase of the wave function of an electron of the lower beam changes by

$$
\Theta_{2}=e \int_{t_{1}}^{t_{2}} d t A_{0}^{(2)}(t),
$$

where $A_{0}^{(2)}$ is the electric field potential along the lower trajectory. It is clear that the phase difference for the electrons of the upper and lower beams $\Theta_{\mathrm{AB}}=\Theta_{2}-\Theta_{1}$ can be written in the form of the integral

$$
\Theta_{\mathrm{AB}}=e \oint_{\gamma} d t A_{0}(t) .
$$

along the closed contour $\gamma$ in the space-time, when the lower half of the contour shown in Fig.1 is passed first and then the upper half is passed in the opposite direction. Figure [1 shows the projection of the contour $\gamma$ onto the space plane.

Let us return to the geometric interpretation. The electron phase difference is given by integral (86), which is unambiguously defined by the contour $\gamma$ and the potential $A_{0}$ set on it. The electric potential $A_{0}$ is the time component of the local $\mathbb{U}(1)$-connection form on the principal fiber bundle $\mathbb{P}\left(\mathbb{R}^{4}, \pi, \mathbb{U}(1)\right)$. Therefore, phase difference (8) defines the element of the holonomy group $\mathrm{e}^{i \Theta_{\mathrm{AB}}} \in \Phi\left(\left(t_{1}, x_{1}\right), e\right) \subset \mathbb{U}(1)$ at the point $\left(t_{1}, x_{1}\right)$, where $x_{1}$ is the space point where the beam is split and $e$ is the unity of the group. The base of the principal fiber bundle $\mathbb{P}$ has trivial topology. Therefore, the contour $\gamma$ can be always contracted to a point, metallic tubes being at their location, and the contour continuously 
passing through them. For contracting contour, the Aharonov-Bohm phase $\Theta_{\text {AB }}$ goes to zero, and the respective element of the holonomy group goes to unity $e \in \mathbb{U}(1)$. This is well known, because the holonomy group is the Lie subgroup in the structure group. We note that contraction of the contour is not related to the experiment where the definite element of the holonomy group corresponding to the initial location of the contour is observed.

The proposed geometric interpretation is not unique. Since the support of the wave function is supposed to be located in a small neighborhood of the trajectory, many principal fiber bundles $\mathbb{P}^{\prime}(\mathbb{M}, \pi, \mathbb{U}(1))$ with different bases $\mathbb{M}$ can be constructed. To this end, it is sufficient to take the fiber bundle $\mathbb{P}\left(\mathbb{R}^{4}, \pi, \mathbb{U}(1)\right)$ with a given connection and to narrow down the base by removing any arbitrary number of arbitrary located straight lines in space $\mathbb{R}^{3}$ which are perpendicular to the plane of Fig.1 and lie outside of the contour $\gamma$. As a result, the base $\mathbb{M}$ becomes not simply connected manifold. The given procedure appears to be unnatural. Moreover, it results in a much more complicated problem since initial Schrödinger equation (44) in that case must be solved on a topologically nontrivial manifold.

At the end of this section, we consider the transformation of the local $\mathbb{U}(1)$-connection form under changing of the section. From Schrödinger equation (5) it follows that under the vertical automorphism

$$
\psi^{\prime}=\mathrm{e}^{i a} \psi
$$

where $a=a(t)$ is a differentiable function of time, components of the local $\mathbb{U}(1)$-connection form are transformed according to the rule

$$
e A_{0}^{\prime}=e A_{0}+\dot{a}
$$

as components of the local $\mathbb{U}(1)$-connection form.

Thus we see that the Aharonov-Bohm effect and the Berry phase are based on the nontrivial geometry, i.e., on the connection with nontrivial holonomy group rather than on the topology. In this case, the space topology can be both trivial and nontrivial.

\subsection{Magnetic potential}

Now we consider the case when the electric field potential is equal to zero, $A_{0}=0$. Suppose that the magnetic field potential depends only on space coordinates $x^{\mu}$ and is independent on time $t$ (a static field). Then the Schrödinger equation takes the form

$$
\begin{aligned}
i \dot{\psi} & =\frac{1}{2 m} \eta^{\mu \nu}\left(\partial_{\mu}+i e A_{\mu}\right)\left(\partial_{\nu}+i e A_{\nu}\right) \psi+U \psi \\
& =\frac{1}{2 m} \eta^{\mu \nu}\left(\partial_{\mu \nu}^{2} \psi+2 i e A_{\mu} \partial_{\nu} \psi+i e \partial_{\mu} A_{\nu} \psi-e^{2} A_{\mu} A_{\nu} \psi\right)+U \psi
\end{aligned}
$$

Let $\phi$ be a solution of the Schrödinger equation in the absence of electromagnetic field (6). Then it is not difficult to verify that the function

$$
\psi=\mathrm{e}^{-i \Theta} \phi
$$

where the phase $\Theta$ satisfies the equation

$$
\partial_{\mu} \Theta=e A_{\mu},
$$

is a solution of initial Schrödinger equation (9). 
Aharonov and Bohm proposed the experiment for the determination of the phase $\Theta$ whose scheme is shown in Fig,2. In this experiment, a beam of electrons is split into two beams bending from different sides an infinitely long solenoid with constant magnetic flux $\Phi$, which is perpendicular to the plane of the figure. Then beams are collected, and the interference pattern depending on the phase difference of electrons in different beams is observed.

To estimate the phase difference of electrons, we make the same assumptions as in the case of the electric field; namely, we consider that the Schrödinger equation without magnetic potential has a solution with the support which is concentrated in a small neighborhood of the electron trajectory. We suppose that this property can be provided by introduction of the appropriate potential in Eq.(6). This potential does not change the topology of space-time, but only ensures motion of electrons along the given trajectory. Then Eqs.(10) are fulfilled for the phase of the solution of the Schrödinger equation with magnetic potential. Since the magnetic field vanishes outside the solenoid, $\partial_{\mu} A_{\nu}-\partial_{\nu} A_{\mu}=$ 0 , the integrability conditions for system of equations (10) are fulfilled. Therefore, the phase difference can be expressed through the contour integral

$$
\Theta_{\mathrm{AB}}=e \oint_{\gamma} d x^{\mu} A_{\mu}
$$

where $\gamma$ is a closed contour in four-dimensional space-time surrounding the solenoid. We note that the term $d x^{0} A_{0}$ in the integrand is equal to zero because $A_{0}=0$ by assumption. This integral is independent of the chosen contour surrounding the solenoid, because the magnetic field outside of the solenoid vanishes.

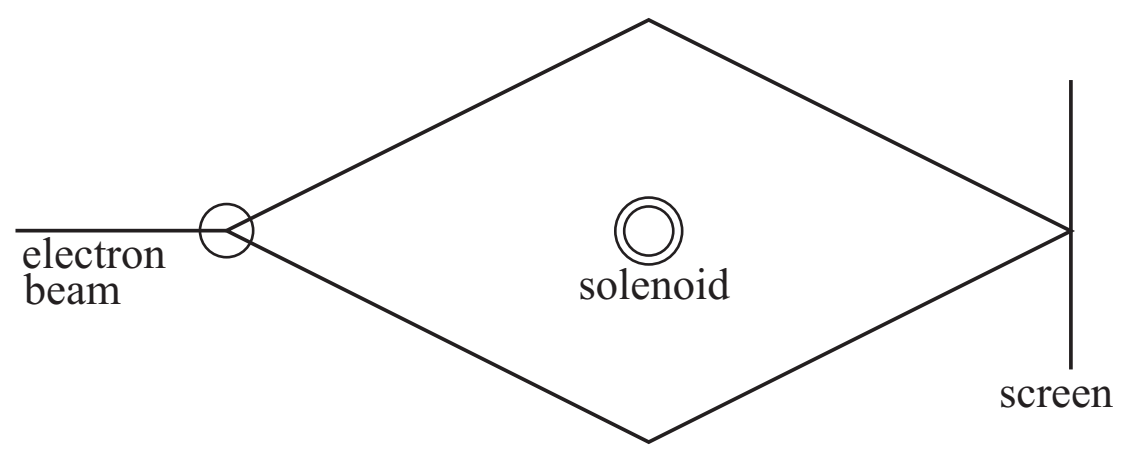

Figure 2: Scheme of the Aharonov-Bohm experiment for the determination of the phase $\Theta$.

The Aharonov-Bohm phase, using the Stokes formulae, can be written in the form of the surface integral

$$
\Theta_{\mathrm{AB}}=\frac{1}{2} e \iint_{S} d x^{\mu} \wedge d x^{\nu} F_{\mu \nu}=e \Phi,
$$

where $F_{\mu \nu}$ is the magnetic field strength (components of the local curvature 2 -form) and $\Phi$ is the total magnetic flux through the solenoid. We note that to use the Stokes formulae, we must consider that the magnetic field is defined everywhere in space $\mathbb{R}^{3}$, including the solenoid itself.

The geometric interpretation of the Aharonov-Bohm effect consists in the following. We have the same principal fiber bundle as in the case of electric potential, $\mathbb{P}\left(\mathbb{R}^{4}, \pi, \mathbb{U}(1)\right)$, whose base is the four-dimensional Euclidean space $(t, x) \in \mathbb{R} \times \mathbb{R}^{3}=\mathbb{R}^{4}$, in which electrons 
move and the structure group is the unitary group $\mathbb{U}(1)$ (the phase factor $\mathrm{e}^{i \Theta}$ of the wave function). However, the connection is now different: only space components of the local connection form $A_{\mu}, \mu=1,2,3$, differ from zero. Aharonov-Bohm phase difference (11) is uniquely defined by the contour $\gamma$ (the same as for the electric potential) and values of the connection form $A_{\mu}$ on it. Writing the contour integral in the form of surface integral $(12)$, we assume that the connection is defined on the whole space-time $\mathbb{R}^{4}$. This means that we consider solenoid of finite radius to avoid singularities.

Thus, the principal fiber bundle is trivial, and the Aharonov-Bohm phase $\Theta_{\mathrm{AB}}$, depending on the connection and the contour, defines uniquely the element of the holonomy group. When the contour is contracted to a point, the corresponding element of the holonomy group goes to unity $e \in \mathbb{U}(1)$ as it must be. Here we assume that the contour goes freely through the solenoid.

As for the electric potential, the electron wave function is the cross section of the associated fiber bundle $\mathbb{E}\left(\mathbb{R}^{4}, \pi_{\mathbb{E}}, \mathbb{C}, \mathbb{U}(1), \mathbb{P}\right)$ with the Euclidean space $\mathbb{R}^{4}$ as the base, and the structure group is the unitary group $\mathbb{U}(1)$. Under the vertical automorphism,

$$
\psi^{\prime}=\mathrm{e}^{i a} \psi
$$

where $a=a(x)$ is a differentiable function of spatial coordinates $x^{\mu}, \mu=1,2,3$, and the magnetic field potential is transformed according to the rule

$$
e A_{\mu}^{\prime}=e A_{\mu}-\partial_{\mu} a
$$

This follows from Schrödinger equation (91). Thus, components of the magnetic field potential have indeed the behavior of components of the local connection form.

Since electron phase difference (11) is defined by values of the local connection form only near the integration contour, the base of the trivial principal fiber bundle $\mathbb{P}\left(\mathbb{R}^{4}, \pi, \mathbb{U}(1)\right)$ can be narrowed down without modification of the answer. For example, we can cut out the domain of the space-time which lies inside the contour $\gamma$ and contains the solenoid. Then the base in no longer simply connected. For this reason the Aharonov-Bohm effect is often called topological. As already demonstrated above, this is not necessary. It is sufficient to assume that the magnetic field differs from zero only on a finite domain on the plain in Fig,2 inside the integration contour. If we assume that the base of the fiber bundle is the Euclidean space $\mathbb{R}^{4}$ with the solenoid cut out, this will create additional difficulties, because the Schrödinger equation must be solved on not simply connected manifold. Moreover, the Stokes formulae cannot be used in such case. Thus, the Aharonov-Bohm effect induced by the magnetic potential must be considered as geometric rather then topological.

The Aharonov-Bohm effect with electric and magnetic potentials attracts great attention of physicists for the following reason. According to contemporary points of view, the gauge-invariant functions are the only observables in gauge models. From this point of view, the electromagnetic field potential $A_{\alpha}, \alpha=0,1,2,3$, is not observable itself, because it is not gauge-invariant. In the considered cases, the electron beams are not subjected to the action of electromagnetic forces, since the electric and magnetic field strengths are zero in the regions where electrons move. Therefore, it seems that the difference in phases of electron beams has to be zero. However, from the Schrödinger equation it follows that it is not so. It should be noted that the electromagnetic field potential is not observable, but we observe its integral along the closed contour which defines the element of the holonomy group of the $\mathbb{U}(1)$-connection being a gauge-invariant object, because the gauge group is Abelian one. 


\section{Conclusions}

In this paper, we have given the geometric interpretation of the Aharonov-Bohm effect. It was demonstrated that the space-time topology in this case can be trivial. Therefore, the Aharonov-Bohm effect, like the Berry phase, has the geometric rather then topological nature.

The interpretation proposed in the paper contains nothing except standard differential geometric notions. In a geometric interpretation of mathematical physics models, one has to take into account that a connection exists on any principal fiber bundle independently of the base topology [9]. Moreover, if a family of local connection forms is given on an arbitrary closed submanifold of the base of some principal fiber bundle, then the corresponding connection can always be continued to the whole principal fiber bundle. This can be done in many ways. The connection defines the holonomy group which generally is nontrivial.

In experiments for measuring the Aharonov-Bohm effect, the observed effects are produced not by the whole holonomy group, but a fixed element of the holonomy group which depends on the connection and the closed contour. The base topology may be trivial or not, it does not play any role. If the topology is trivial, the integration contour can be contracted to a point. The effect disappears in this case because the corresponding element of the holonomy group goes to the unity element, and this is quite natural from the physical viewpoint.

A connection on the principal fiber bundle defines connections on all fiber bundles which are associated with it. In particular, if the typical fiber is an infinitely dimensional Hilbert space, then the connection is also defined. At present, the interpretation of the Berry phase and the Aharonov-Bohm effect, as a rule, is reduced to consideration of a connection on an associated fiber bundle, and this forces one to consider infinitely dimensional manifolds and to take into account the related subtleties. From our point of view, the interpretation of geometric effects in terms of connections on principal fiber bundles is simpler and more natural.

The author is grateful to I. V. Volovich for discussions and fruitful comments.

This work was supported in part by the Russian Foundation for Basic Research (grants 11-01-00828-a and 11-01-12114-ofi_m), the Program for Supporting Leading Scientific Schools (Grant No. NSh-7675.2010.1), and the program "Contemporary problems of theoretical mathematics" of the Russian Academy of Sciences.

\section{References}

[1] Y. Aharonov and D. Bohm. Significance of electromagnetic potentials in the quantum theory. Phys. Rev., 115(3):485-491, 1959.

[2] F. G. Werner and D. R. Brill. Significance of electromagnetic potentials on the quantum theory in the interpretation of electron interferometer fringe observations. Phys. Rev. Lett., 4(7):344-347, 1960.

[3] R. G. Chambers. Shift of an electron interference pattern by enclosed magnetic flux. Phys. Rev. Lett., 5(1):3-5, 1960.

[4] H. Boersch, H. Hamisch, D. Wohlleben, and K. Grohmann. Weissche bereiche als bi-prisme für elektroneninterferenzen. Z. Phys., 159:397-404, 1960. 
[5] M. V. Berry. Quantal phase factors accompanying adiabatic changes. Proc. Roy. Soc. London, A392(1802):45-57, 1984.

[6] M. O. Katanaev. On geometric interpretation of the Berry phase. Izv. vuzov. Phys. To be published.

[7] E. Schrödinger. Quantizierung als Eigenwertproblem (Erste Mitteilung). Ann. Phys. Leipzig, 79(4):361-376, 1926.

[8] E. Schrödinger. Quantizierung als Eigenwertproblem (Zweite Mitteilung). Ann. Phys. Leipzig, 79(6):489-527, 1926.

[9] S. Kobayashi and K. Nomizu. Foundations of differential geometry, volume 1, 2. Interscience publishers, New York - London, 1963. 